\title{
MOOCs sobre tecnologia assistiva no contexto educacional: uma revisão sistemática
}

Bruna Poletto Salton, IFRS Campus Porto Alegre, bruna.salton@ifrs.edu.br Silvia Bertagnolli, IFRS Campus Porto Alegre, silvia.bertagnolli@ poa.ifrs.edu.br

Resumo. Para muitos estudantes com deficiência, o acesso a recursos e serviços de Tecnologia Assistiva (TA) pode ser o diferencial para que tenham autonomia e êxito no contexto educacional. Para viabilizar essa realidade, há demanda crescente por capacitação em TA que chegue até os envolvidos nesse processo, de forma ampla, aberta e gratuita, características estas que podem ser contempladas pela oferta de Massive Open Online Courses (MOOCs). Nesse sentido, foi conduzida uma revisão sistemática para prover uma visão geral do estado corrente da pesquisa relacionada à utilização de MOOCs para oferecer capacitação em TA no contexto educacional. Como resultados, verificouse a quase inexistência de MOOCs sobre TA e que o tema ainda é pouco explorado, mas com grande potencial para atender uma demanda urgente e crescente no contexto educacional.

Palavras-chave. Tecnologia Assistiva, MOOCs, Contexto Educacional.

Abstract. For many students with disabilities, having access to Assistive Technology (AT) resources and services can be an essential aspect for them to have autonomy and success in the educational context. To make this reality possible, there is a growing demand for training in AT that reaches those involved in this process, in a broad, open and free manner, characteristics that can be contemplated by the offer of Massive Open Online Courses (MOOCs). In this sense, a systematic review was conducted to provide an overview of the current state of research related to the use of MOOCs to offer training in AT in the educational context. As a result, there was almost no MOOCs on AT and the topic is still little explored, but with great potential to meet an urgent and growing demand in the educational context.

Keywords. Assistive Technology, MOOCs, Educational Context.

\section{Introdução}

A Tecnologia Assistiva (TA) abrange recursos e serviços que proporcionam maior autonomia e independência às pessoas com deficiência (Galvão Filho, 2009). Na educação, a TA se faz essencial no processo de inclusão de estudantes com deficiência, de modo que, para muitos deles, ter acesso a recursos e serviços de TA adequados pode ser o diferencial para o êxito escolar. No entanto, por mais que se incentive a utilização da TA para oferecer possibilidades de participação e autonomia aos estudantes com deficiência, a lacuna na área de formação de recursos humanos nessa área é enorme (Calheiros; Mendes; Lourenço, 2018).

Considerando a necessidade de capacitação em TA, os Massive Open Online Courses (MOOCs) parecem ser uma possibilidade interessante, oferecendo soluções amplamente disponíveis, gratuitas e que possam ser realizadas a qualquer tempo. Esses cursos online abertos e massivos possuem acesso aberto, conteúdo instrucional global, gratuito, com flexibilidade de horário e local; e baseado em vídeos, leituras, integração com redes sociais, fóruns e outras estratégias que podem ser gerenciadas através de uma plataforma online (Baturay, 2015).

Nesse sentido, uma revisão sistemática foi conduzida com o objetivo de prover uma visão geral do estado corrente da pesquisa relacionada à utilização de MOOCs para oferecer capacitação na área de Tecnologia Assistiva (TA) no contexto educacional. V. $18 \mathrm{~N}^{\mathrm{o}} 1$, julho, 2020 RENOTE DOI: 
Procurou-se, também, investigar pontos específicos a respeito dos MOOCs oferecidos com esse propósito, quais benefícios e fragilidades apresentam, quais metodologias, estratégias, teorias e tecnologias foram utilizadas em sua estruturação.

A partir dos resultados obtidos com o presente mapeamento, espera-se identificar a viabilidade e efetividade de utilização de MOOCs para oferecer capacitação em TA no contexto educacional.

\section{Tecnologia Assistiva no contexto educacional e MOOCs}

A inclusão no sistema regular de ensino é um direito assegurado pela legislação brasileira. Nesse sentido, a Lei Brasileira de Inclusão da Pessoa com Deficiência (LBI) reforça que as instituições de ensino devem garantir "condições de acesso, permanência, participação e aprendizagem, por meio da oferta de serviços e de recursos de acessibilidade que eliminem as barreiras e promovam a inclusão plena" (Brasil, 2015, p. 07). Nesse contexto, a Tecnologia Assistiva amplia as possibilidades de estudantes com deficiência, lhes oferecendo autonomia e independência. Para muitos desses estudantes, receber o apoio necessário quanto à escolha e uso dos recursos de TA adequados é essencial para seu êxito escolar.

O número de matrículas de estudantes com deficiência chegou a 1,2 milhão em 2018, um aumento de 33,2\% em relação a 2014 (Inep, 2019). Igualmente crescente é a necessidade de ter profissionais preparados para atender esses alunos, inclusive no que tange ao uso da Tecnologia Assistiva. "A abrangência do conceito garante que TA não se restringe somente a recursos em sala de aula, mas estende-se a todos os ambientes da escola, propiciando o acesso e a participação efetiva de todos os alunos e durante todo o tempo" (Its Brasil, 2008, p.11). Isso quer dizer que toda a comunidade acadêmica deveria ser co-responsável nesse processo, trazendo à tona a necessidade de capacitação em TA no contexto educacional.

Estudos como os de Emer, Mauer e Batecini (2014) e Borges e Tartuci (2017), que buscaram identificar o nível de conhecimento que professores possuem sobre TA, têm apontado que este é um tema ainda pouco difundido entre os docentes, mesmo entre aqueles que atuam no Atendimento Educacional Especializado (AEE). Já a investigação de Calheiros e Mendes (2016) aborda iniciativas do Ministério da Educação (MEC) visando a formação de professores para atuar no AEE. Segundo esses autores, tais iniciativas têm sido em nível de aperfeiçoamento e de pós-graduação lato sensu, nas quais a TA é apenas uma pequena parte dentre vários conteúdos e abordada de forma essencialmente teórica, causando poucas mudanças nas práticas pedagógicas voltadas aos estudantes com deficiência.

Considerando, portanto, o número crescente de estudantes com deficiência na rede regular de ensino, a importância da TA para o êxito escolar destes estudantes e a necessidade de haver capacitação nessa área, os MOOCs representam possibilidades abrangentes e acessíveis para suprir essa demanda.

Os MOOCs são cursos online, abertos, ou seja, sem restrição geográfica, financeira, de idade ou escolaridade (Clair et al., 2015) e massivos, já que milhares de pessoas podem fazer um curso simultaneamente, sendo esta a característica que os difere de outras experiências de e-learning (Sánchez-Vera; Leon; Davis, 2015). Além disso, é interessante considerar o tamanho da rede e a capacidade de gerar novos conhecimentos dos MOOCs, refletindo a aprendizagem participativa e respeitando a diversidade entre o grande número de alunos existentes (Knox; Bayne, 2014).

Alinhando-se a necessidade de oferecer capacitação abrangente em TA no contexto educacional às possibilidades trazidas pelos MOOCs nesse sentido, uma revisão 
sistemática de literatura a esse respeito pode trazer dados importantes sobre iniciativas dessa natureza e suas especificidades.

\section{Metodologia}

Um estudo primário é um estudo empírico que investiga uma questão de pesquisa específica, ao passo que um estudo secundário é o que analisa todos os estudos primários relacionados a uma questão de pesquisa específica com o objetivo de integrar e sintetizar evidências relacionadas à questão de pesquisa. Uma revisão sistemática é uma forma de estudo secundário que utiliza uma metodologia bem definida para identificar, analisar e interpretar os dados disponíveis a respeito de uma questão de pesquisa (Kitchenham; Charters, 2007). Através de uma revisão sistemática, é possível construir uma síntese de estudos científicos de qualidade a partir de uma revisão metodologicamente rigorosa dos resultados da investigação (Kitchenham et al, 2009). O principal objetivo é identificar lacunas que podem ser abordadas em investigações futuras.

As próximas seções apresentam os passos da revisão sistemática, iniciando pela definição das questões de pesquisa, passando à condução da busca em bases de dados pré-definidas, apresentação dos resultados a partir de critérios de inclusão e exclusão e extração e análise de dados, de acordo com o que é sugerido por Petersen et al. (2008).

\subsection{Definição das questões de pesquisa}

Especificar as questões de pesquisa é a parte mais importante da revisão sistemática, pois são essas questões que orientam toda a metodologia (Kitchenham; Charters, 2007). Desse modo, iniciou-se esse processo definindo as questões de pesquisa, as quais são apresentadas no Quadro 1.

Quadro 1: Questões de pesquisa

\begin{tabular}{|l|l|}
\hline QP1 & Quando e onde os estudos têm sido publicados? \\
\hline QP2 & Que tipos de pesquisa têm sido feitas? \\
\hline QP3 & Que problemas têm sido apontados? \\
\hline QP4 & Quais os pontos positivos apresentados? \\
\hline QP5 & Quais as fragilidades identificadas? \\
\hline QP6 & Quais metodologias têm sido utilizadas no curso? \\
\hline QP7 & Qual a carga horária total do curso? \\
\hline QP8 & Qual o público alvo do curso? \\
\hline QP9 & Quais tecnologias têm adotadas no curso? \\
\hline QP10 & Que tipos de recursos de TA têm sido abordados no curso? \\
\hline
\end{tabular}

Fonte: elaborado pelos autores, 2020

As questões QP1 e QP2 estão relacionadas ao local e tipo de publicação; QP3 a QP5 buscam informações mais gerais sobre o conteúdo dos estudos; QP6 a QP10 indagam a respeito de pontos específicos dos cursos que são abordados nos estudos em análise. 


\subsection{Condução da busca}

A condução da busca iniciou com a definição dos termos a serem pesquisados. Assim, foram selecionados os termos "tecnologia assistiva" e "ajuda técnica", pois aparecem como sinônimos na legislação brasileira; já a expressão "tecnologia de apoio" foi incluído na chave de busca, porque é o termo equivalente utilizado em Portugal. Como a investigação será conduzida por bases de dados internacionais foram selecionados os termos "assistive technology" e "adaptative technology" os quais são comumente utilizados na língua inglesa para referir-se à Tecnologia Assistiva. Assim, para a revisão sistemática, utilizou-se a string de busca ilustrada pela Figura 1.

Figura 1: String de busca utilizada

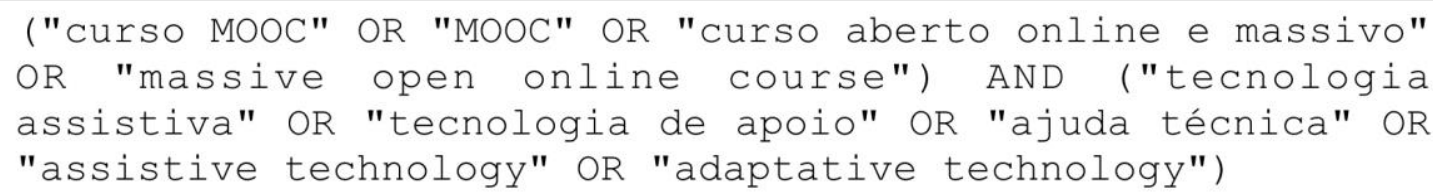

Fonte: elaborado pelos autores, 2020

Optou-se por delimitar a pesquisa em apenas dois termos, os cursos MOOC e a Tecnologia Assistiva, para não correr o risco de não se ter resultados suficientes. Nessa chave de busca foi incluída a expressão curso MOOC e suas derivações, visando ampliar os resultados obtidos com a pesquisa. As investigações foram realizadas no Portal de Periódicos CAPES, Portal de Teses e Dissertações, Portal de Publicações da Comissão Especial de Informática na Educação (CEIE), Revista de Novas Tecnologias na Educação (RENOTE) e no Journal of Special Education. Apesar de o termo MOOC ter surgido em 2008, a busca limitou-se a artigos publicados a partir de 2012, ano em que esses cursos começaram a ganhar popularidade, e período em que as plataformas Coursera, Udacity e Udemy (Baturay, 2015) passam a ter mais visibilidade.

\subsection{Resultados da busca}

Para a apresentação dos resultados da busca foi definido como único critério de inclusão estudos cujo tema esteja relacionado a MOOCs e Tecnologia Assistiva. Já como critérios de exclusão foram definidos os apresentados no Quadro 2.

Quadro 2: Critérios de inclusão e exclusão de estudos identificados

\begin{tabular}{|l|l|}
\hline Critério de inclusão & Critérios de exclusão \\
\hline CI - O estudo aborda tema relacionado a & CE1 - O estudo não tem resumo \\
MOOCs e Tecnologia Assistiva & CE2 - O estudo não é um estudo primário \\
CE3 - Não foi possível ter acesso ao texto completo \\
do estudo \\
CE4 - O estudo tem como foco o uso de TA para \\
realizar um curso MOOC \\
CE5 - O estudo tem como foco a acessibilidade em \\
curso MOOC
\end{tabular}

Fonte: elaborado pelos autores, 2020

Como o objetivo deste estudo é investigar o uso de MOOCs para oferecer capacitação em TA, os critérios de exclusão 4 e 5 foram estabelecidos para que possíveis resultados envolvendo MOOCs e TA em um contexto diferente não fossem incluídos para a etapa de análise, visto que esse não é foco da investigação. Por exemplo, realizar um 
MOOC com leitor de tela ou com um mouse adaptado, ou verificar a acessibilidade em MOOCs não poderiam ser incluídos na análise conduzida por este trabalho.

Como resultado da busca, foram obtidos 17 estudos. Destes, 35\% não abordavam o tema MOOCs e Tecnologia Assistiva, sendo eliminados já nesse primeiro momento de seleção das pesquisas; e considerando os outros $65 \%$ que abordavam a temática, nove pesquisas foram eliminadas pelos critérios de exclusão (CE1, CE2 ou CE5) e duas incluídas para análise

O Quadro 3 apresenta o detalhamento dos artigos incluídos e excluídos. Nota-se que este quadro descreve a origem do artigo (base onde foi publicado), o ano em que o mesmo foi publicado, o seu título, os critérios de exclusão (com base nos itens elencados no Quadro 3), e o critério de inclusão descrito anteriormente.

Quadro 3: Estudos Identificados x Critérios Aplicados

\begin{tabular}{|c|c|c|c|c|}
\hline Base & Ano & Título & $\begin{array}{l}\text { Excluir } \\
\text { critério(s) }\end{array}$ & Incluir \\
\hline $\begin{array}{l}\text { Directory of } \\
\text { Open Access } \\
\text { Journals } \\
(\text { DOAJ })^{1}\end{array}$ & 2018 & $\begin{array}{l}\text { Tecnologias e ferramentas para elaboração de conteúdos em } \\
\text { um ambiente MOOC: estudo de caso a partir de uma formação } \\
\text { em Tecnologias Assistivas (FRANSCISCATTO et al., 2018) }\end{array}$ & & $\mathrm{X}$ \\
\hline $\begin{array}{l}\text { ERIC (U.S. } \\
\text { Dept. of } \\
\text { Education) }\end{array}$ & 2013 & $\begin{array}{l}\text { MOOCs: When Opening Doors to Education, Institutions Must } \\
\text { Ensure That People with Disabilities Have Equal Access }\end{array}$ & CE5 & \\
\hline Library Journal & 2018 & $\begin{array}{l}\text { Accessibility on Campus: Legal requirements, the growth of } \\
\text { dedicated positions, and enhanced technology are helping } \\
\text { academic libraries make content accessible for all }\end{array}$ & CE1 & \\
\hline $\begin{array}{l}\text { ProQuest } \\
\text { Computing } \\
\text { Database }\end{array}$ & 2019 & $\begin{array}{l}\text { Propuesta de aplicación de minería de procesos para evaluar } \\
\text { las rutas de aprendizaje de estudiantes con discapacidad visual } \\
\text { en cursos en línea }\end{array}$ & CE5 & \\
\hline $\begin{array}{l}\text { ACM Digital } \\
\text { Library }\end{array}$ & 2018 & $\begin{array}{l}\text { Research challenges in accessible MOOCs: a systematic } \\
\text { literature review } 2008-2016\end{array}$ & CE2, CE5 & \\
\hline SpringerLink & 2018 & $\begin{array}{l}\text { The problems disabled people face in mobile and web based e- } \\
\text { learning phases in a developing country }\end{array}$ & CE5 & \\
\hline SpringerLink & 2018 & $\begin{array}{l}\text { The Case for Captioned Lectures in Australian Higher } \\
\text { Education }\end{array}$ & CE5 & \\
\hline $\begin{array}{l}\text { ProQuest } \\
\text { Computing } \\
\text { Database }\end{array}$ & 2017 & More Than Enough Is Too Much & CE5 & \\
\hline ScienceDirect & 2016 & $\begin{array}{l}\text { Balancing pedagogy, student readiness and accessibility: A } \\
\text { case study in collaborative online course development }\end{array}$ & CE5 & \\
\hline ScienceDirect & 2019 & $\begin{array}{l}\text { Online e-learning and cognitive disabilities: A systematic } \\
\text { review }\end{array}$ & CE2 & \\
\hline $\begin{array}{l}\text { Portal de } \\
\text { Publicações da } \\
\text { CEIE }\end{array}$ & 2017 & $\begin{array}{l}\text { SolAssist Learning: formação em tecnologias assistivas através } \\
\text { de um MOOC e uma biblioteca virtual de soluções assistivas } \\
\text { (WAGNER et al., 2017) }\end{array}$ & & $\mathrm{X}$ \\
\hline
\end{tabular}

Fonte: elaborado pelos autores, 2020

\footnotetext{
${ }^{1}$ Busca realizada a partir do Portal de Periódicos da CAPES (Coordenação de Aperfeiçoamento de Pessoal de Nível Superior)

V. I8 $\mathrm{N}^{\mathrm{N}} 1$, julho, 2020 RENOTE DOI:
} 
Após aplicar os critérios de exclusão, obteve-se, então, como relevante para o propósito desta pesquisa, apenas dois estudos nacionais: Franciscatto et al. (2018), chamado de Estudo 1, e Wagner et al. (2017), chamado de Estudo 2, onde ambos abordam o curso SolAssist Learning.

\subsection{Análise dos resultados}

A análise dos estudos foi conduzida a partir das questões de pesquisa apresentadas no Quadro 1. Considerando que ambos os estudos incluídos para a etapa de análise tratam do mesmo curso MOOC, o SolAssist Learning, muitas respostas se aplicam a ambos os estudos. As informações resultantes da análise desses trabalhos, usando as questões de pesquisa do Quadro 1, encontram-se detalhadas no Quadro 4.

Quadro 4: Detalhamento dos artigos

\begin{tabular}{|c|c|}
\hline QP1 & Quando e onde os estudos têm sido publicados? \\
\hline Estudo 1 & 2018 - Revista Observatório ${ }^{2}$ \\
\hline Estudo 2 & 2017 - Informática da Educação: Teoria e Prática ${ }^{3}$ \\
\hline QP2 & Que tipos de pesquisa têm sido feitas? \\
\hline Estudo 1 & Estudo de caso \\
\hline Estudo 2 & Proposta de modelo de curso e ferramenta \\
\hline QP3 & Que problemas têm sido apontados? \\
\hline $\begin{array}{l}\text { Estudo } 1 \\
\text { Estudo } 2\end{array}$ & $\begin{array}{l}\text { Necessidade de capacitação de professores do ensino profissionalizante que atuam na } \\
\text { preparação para a inclusão de pessoas com deficiência no mercado de trabalho. }\end{array}$ \\
\hline QP4 & Quais os pontos positivos apresentados? \\
\hline $\begin{array}{l}\text { Estudo } 1 \\
\text { Estudo } 2\end{array}$ & $\begin{array}{l}\text { O poder de interação e colaboração dos MOOCs em conjunto com uma biblioteca de } \\
\text { soluções assistivas que provê um sistema hierárquico dinâmico e prático ao aprendizado } \\
\text { sobre as TAs. }\end{array}$ \\
\hline QP5 & Quais as fragilidades identificadas? \\
\hline $\begin{array}{l}\text { Estudo } 1 \\
\text { Estudo } 2\end{array}$ & $\begin{array}{l}\text { Participantes selecionados por indicação } \\
\text { Uso de ferramentas com interface em inglês } \\
\text { Dos } 34 \text { selecionados, } 10 \text { concluíram o curso com sucesso }\end{array}$ \\
\hline QP6 & Quais metodologias têm sido utilizadas no curso? \\
\hline $\begin{array}{l}\text { Estudo } 1 \\
\text { Estudo } 2\end{array}$ & $\begin{array}{l}\text { Curso organizado em sete módulos, distribuídos em } 14 \text { semanas } \\
\text { Maior parte das atividades foi desenvolvida no próprio ambiente de trabalho dos } \\
\text { participantes } \\
\text { Interação baseada no uso de fóruns e questionários } \\
\text { Biblioteca virtual de soluções assistivas como parte do curso, para ampliar o acesso a } \\
\text { recursos de TA pelos participantes }\end{array}$ \\
\hline QP7 & Qual a carga horária total do curso? \\
\hline $\begin{array}{l}\text { Estudo } 1 \\
\text { Estudo } 2\end{array}$ & $100 \mathrm{~h}$ \\
\hline
\end{tabular}

\footnotetext{
${ }^{2}$ https://sistemas.uft.edu.br/periodicos/index.php/observatorio/index

${ }^{3} \mathrm{https}$ ://seer.ufrgs.br/InfEducTeoriaPratica/index

V. $18 \mathrm{~N}^{\circ} 1$, julho, 2020 


\begin{tabular}{|l|l|}
\hline QP8 & Qual o público alvo do curso? \\
\hline $\begin{array}{l}\text { Estudo 1 } \\
\text { Estudo 2 }\end{array}$ & $\begin{array}{l}\text { Profissionais que atuam em cursos profissionalizantes e com pessoas com deficiência } \\
\text { matriculadas. }\end{array}$ \\
\hline QP9 & Quais tecnologias têm sido utilizadas no curso? \\
\hline $\begin{array}{l}\text { Estudo 1 } \\
\text { Estudo 2 }\end{array}$ & Wikispaces, PbWorks, Google Drive \\
\hline QP10 & Que tipos de recursos de TA têm sido abordados no curso? \\
\hline $\begin{array}{l}\text { Estudo 1 } \\
\text { Estudo 2 }\end{array}$ & Foco em recursos e práticas para a inserção laboral. \\
\hline
\end{tabular}

Fonte: elaborado pelos autores, 2020

Percebe-se que, dentre as fragilidades apontadas (QP5), estão a indicação de participantes e o uso de ferramentas em inglês. Quando os participantes são selecionados por indicação isso acaba reduzindo as chances de conclusão com êxito do curso, visto que o interesse deve motivar o estudante (Zheng, 2016) além de contrariar umas das características essenciais dos MOOCs, o acesso aberto. Quanto às ferramentas com uso do idioma inglês, Zhou (2016) aponta obstáculos linguísticos como um dos fatores que pode desfavorecer a aprendizagem em MOOCs, sendo que conteúdo e/ou ferramentas em um idioma diferente do idioma nativo do estudante podem contribuir para a evasão nesse tipo de curso.

A taxa de conclusão do curso (aproximadamente 29\%) não é incomum em MOOCs, já que muitas pessoas se inscrevem nestes cursos sem ter a intenção de finalizálos, alguns por curiosidade, outros para ter acesso ao material, outros por desconhecerem o alto nível de organização que é necessário, por parte do estudante, para atender às interações que muitos cursos impõem. No entanto, é justamente o acesso aberto e a inexistência de pré-requisitos que encoraja inscrições de pessoas com essas motivações (GROVER et al, 2013), o que não ocorre no curso analisado através dos estudos 1 e 2, o qual apresenta pré-requisitos e conta com participantes pré-selecionados por indicação, ou seja, esperar-se-ia um perfil de participante mais engajado e disposto a finalizar o curso.

Analisando-se a duração do curso (14 semanas), estudos apontam que cursos mais curtos têm um número maior de concluintes. Enquanto cursos de 4 semanas têm taxas de conclusão de 30\%, em cursos com mais de 10 semanas essas taxas são de aproximadamente de $8 \%$ (Jordan, 2015). Acredita-se que este seja um dos fatores que influenciaram o número reduzido de concluintes no curso dos estudos analisados.

Pode-se concluir que, apesar da grande relevância do curso analisado detalhado através dos estudos 1 e 2, o foco é a inclusão das pessoas com deficiência no mercado de trabalho, tendo um público alvo bem específico, bem como o conteúdo abordado. Nesse sentido, pode-se dizer que não foram encontrados estudos relacionados a cursos MOOC sobre TA especificamente no contexto educacional. Essa revisão sistemática deixa clara a escassez de iniciativas de MOOCs para capacitar a comunidade acadêmica de uma instituição para que os alunos com deficiência tenham suas necessidades relacionadas à TA atendidas de forma efetiva, possibilitando-lhes realizar seu percurso acadêmico com autonomia e igualdade de oportunidades. 


\subsection{Busca complementar}

Como complemento à revisão sistemática, já que foi gerado um número bastante reduzido de publicações científicas, foram realizadas pesquisas diretamente em plataformas de cursos MOOC. O termo de busca utilizado foi "tecnologia assistiva", sendo que em plataformas internacionais, utilizou-se, também, o termo "assistive technology".

$\mathrm{Na}$ plataforma Coursera ${ }^{4}$, foram encontrados dois cursos: o curso "An Introduction to Accessibility and Inclusive Design" ${ }^{5}$, no qual a TA é abordada no módulo 1 do curso, de forma básica, não tem foco no contexto educacional e é oferecido somente em idioma inglês; e o curso "Disability Awareness and Support" ${ }^{6}$ que aborda a questão da TA no ensino superior, de forma introdutória, no módulo 2 do curso. De forma semelhante ao curso anterior ele é oferecido somente em inglês. A busca na plataforma Udemy teve como único resultado, um curso de JAWS (Job Access With Speech), denominado "Introdução ao JAWS - tecnologia assistiva para cegos"7, o qual explica como utilizar esse leitor de tela para o sistema operacional Windows. Já na plataforma EdX o único resultado localizado foi o curso "Disability and Digital Media: Accessibility,

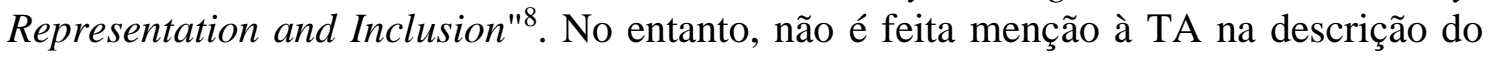
curso, nem no conteúdo programático dos seus módulos.

Ainda, foram realizadas buscas, usando a chave de pesquisa ilustrada pela Figura 1, no Google e obteve-se como resultado, apenas o curso "Tecnologia Assistiva, Projetos e Acessibilidade Promovendo a Inclusão Escolar" ${ }^{\prime \prime}$, da UNESP, o qual aborda a TA apenas no módulo 2, sendo que todo o conteúdo do curso foi compilado em um livro digital em formato PDF. O material deste curso foi feito, inicialmente, para ser utilizado na plataforma TelEduc, e era composto por vídeos que foram desenvolvidos em Flash, formato que está em desuso e já não é suportado por algumas plataformas. Além disso, sobre a TA, o material, basicamente, traz manuais de alguns recursos de acessibilidade do Windows e das ferramentas Teclado Amigo, Lente Pro e DOSVOX.

\section{Considerações finais}

Através desta revisão sistemática, percebe-se que cursos MOOC que abordam a TA são um assunto ainda pouco explorado e, no entanto, muito necessário. Pelo que se pode analisar, não há, atualmente, no Brasil, um curso livre e gratuito, com conteúdo atual, certificado por instituição de ensino ou de pesquisa reconhecida nacionalmente, que investigue o tema com profundidade, para oferecer capacitação em Tecnologia Assistiva aos profissionais da educação. No entanto, a urgência é crescente, pois o ingresso de alunos com os mais variados tipos de deficiência nas instituições de ensino está aumentando a cada ano.

Esta revisão sistemática mostra que há espaço para iniciativas que ofereçam capacitação em TA por meio de MOOCs, na tentativa de preencher a lacuna na formação de pessoas para atuar nessa área no contexto educacional. O estudo traz ainda a necessidade de trabalhos futuros, que tragam discussões a respeito do conteúdo, recursos e abordagens para que os MOOCs sejam espaços de aprendizagem, com oportunidades de trocas, colaboração e engajamento.

\footnotetext{
${ }^{4}$ https://www.coursera.org

${ }^{5} \mathrm{https} / / /$ pt.coursera.org/learn/accessibility

${ }^{6} \mathrm{https}: / /$ pt.coursera.org/learn/disability-awareness

${ }^{7} \mathrm{https} / / / \mathrm{www}$.udemy.com/course/introducao-ao-jaws-tecnologia-assistiva-para-cegos

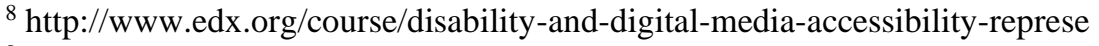

9 https://acervodigital.unesp.br/handle/123456789/47325

V. $18 \mathrm{~N}^{\mathrm{o}} 1$, julho, 2020

DOI: 


\section{Referências}

BATURAY, M. H. An overview of the world of moocs. Procedia-Social and

Behavioral Sciences, v. 174, p. 427-433, 2015. Disponível em:

<https://www.sciencedirect.com/science/article/pii/S1877042815007363?via\%3Dihub> Acesso em: maio 2020.

BORGES, W. F.; TARTUCI, D. Tecnologia Assistiva: Concepções de Professores e as Problematizações Geradas pela Imprecisão Conceitual. Rev. bras. educ. espec., Marília, v. 23, n. 1, p. 81-96, Mar. 2017. Disponível em:

$<$ http://www.scielo.br/scielo.php?pid=S1413-

65382017000100081\&script=sci_abstract\&tlng=pt> Acesso em: maio 2020.

BRASIL. Lei $\mathbf{n}^{0}$ 13.146 de 6 de Julho de 2015. Institui a Lei Brasileira de Inclusão da Pessoa com Deficiência (Estatuto da Pessoa com Deficiência). Brasília, 2015.

Disponível em: http://www.planalto.gov.br/ccivil_03/_Ato2015-

2018/2015/Lei/L13146.htm. Acesso em: mar. 2020.

CALHEIROS, D. S.; MENDES, E. G. Consultoria colaborativa a distância em tecnologia assistiva para professores. Cad. Pesqui., São Paulo, v. 46, n. 162, p. 11001123, out./dez., 2016. Disponível em:

$<$ http://www.scielo.br/scielo.php?script=sci_arttext\&pid=S0100-

15742016000401100\&lng=en\&nrm=iso> Acesso em: abr. 2020.

CALHEIROS, D. S.; MENDES, E. G. LOURENÇO, G. F. Considerações acerca da tecnologia assistiva no cenário educacional brasileiro. Revista Educação Especial, v. 31, n. 60, jan./mar. 2018. Disponível em:

<https://periodicos.ufsm.br/educacaoespecial/article/view/18825/pdf> Acesso em: abr. 2020.

CLAIR, R. S., WINER, L., FINKELSTEIN, A., WALD, S., FINKELSTEIN, A., FUENTES-STEEVES, A. (2015). Big Hat and No Cattle? The implications of MOOCs for the adult learning landscape. The Canadian Journal for the Study of Adult Education, v. 27, n. 3, p. 65-82. Disponível em: <https://eric.ed.gov/?id=EJ1186658> Acesso em: mar. 2020.

EMER, S. O.; MAUER, J. L.; BATECINI, L. Formação docente para o uso da tecnologia assistiva na educação infantil. RENOTE - Revista Novas Tecnologias na Educação. v. 12, n. 2, dezembro, 2014. Disponível em: <https://seer.ufrgs.br/renote/article/view/53558/33057> Acesso em: maio 2020.

FRANCISCATTO, R.; WAGNER, R.; PASSERINO, L. M. Tecnologias e ferramentas para elaboração de conteúdos em um ambiente MOOC: estudo de caso a partir de uma formação em Tecnologias Assistivas. Revista Observatório, v.4, n.3, p. 361-398, 29 abr. 2018. Disponível em:

<https://sistemas.uft.edu.br/periodicos/index.php/observatorio/article/view/4077> Acesso em: mar. 2020.

GALVÃO FILHO, T. A. A Tecnologia Assistiva: de que se trata? In: MACHADO, G. J. C.; SOBRAL, M. N. (Orgs.). Conexões: educação, comunicação, inclusão e interculturalidade. 1 ed. Porto Alegre: Redes Editora, p. 207-235, 2009.

GROVER, S.; FRANZ, P.; SCHNEIDER, E.; PEA, R. The MOOC as distributed intelligence: dimension of a framework \& evaluation of MOOCs. ComputerSupported Collaborative Learning Conference, CSCL, 2013. Disponível em: $<$ https://web.stanford.edu/ shuchig/docs/Framework\%20for\%20Design\%20\&\%20Eval uation\%20of\%20MOOCs-Grover-Franz-Schneider-Pea.pdf> Acesso em: maio 2020. 
INEP. Notas estatísticas: Censo Escolar 2018. Inep/MEC, Brasília, 2019. Disponível em:

<http://download.inep.gov.br/educacao_basica/censo_escolar/notas_estatisticas/2018/no tas_estatisticas_censo_escolar_2018.pdf $>$ Acesso em: maio 2020.

ITS BRASIL. Tecnologia Assistiva nas Escolas: recursos básicos de acessibilidade sócio-digital para pessoas com deficiência. Instituto de Tecnologia Social, 2008.

Disponível em:

<https://docs.wixstatic.com/ugd/85fd89_a9baa902e9c94ce5b8b19e4072baf46a.pdf>

Acesso em: mar. 2020.

JORDAN, K. MOOC Completion Rates: The Data. 2015. Disponível em: http://www.katyjordan.com/MOOCproject.html. Acesso em: maio 2020.

KITCHENHAM, B.; CHARTERS, S. Guidelines for performing systematic

literature reviews in software engineering. Technical Report EBSE 2007-001, Disponível em:

<https://www.elsevier.com/_data/promis_misc/525444systematicreviewsguide.pdf> Acesso em: mar. 2020.

KITCHENHAM, B.; BRERETON, P.; BUDGEN, D.; TURNER, M.; BAILEY, J.; LINKMAN, S. Systematic literature reviews in software engineering - A systematic literature review. Information and Software Technology, v. 51, p. 7-15, jan. 2009. Disponível em: <https://doi.org/10.1016/j.infsof.2008.09.009> Acesso em: abr. 2020.

KNOX, J.; BAYNE, S. Multimodal profusion in the literacies of the Massive Open Online Course. Research in Learning Technology, v. 21, jan. 2014. Disponível em: <https://journal.alt.ac.uk/index.php/rlt/article/view/1438> Acesso em: abr. 2020.

PETERSEN, K.; FELDT, R.; MUJTABA, S.; MATTSSON, M. Systematic Mapping Studies in Software Engineering. Proceedings International Conference on Evaluation and Assessment in Software Engineering, Italy, p. 68-77, 2008. Disponível em: <https://www.sciencedirect.com/science/article/abs/pii/S0950584915000646> Acesso em: abr. 2020.

SÁNCHEZ-VERA; M.; LEON, M.; DAVIS, H. Challenges in the Creation,

Development and Implementation of MOOCs: Web Science Course at the University of Southampton. Comunicar, v. 22. p. 37-44, jan. 2015. Disponível em: $<$ https://www.revistacomunicar.com/verpdf.php?numero $=44 \&$ articulo=44-201504\&idioma=en $>$ Acesso em: abr. 2020.

WAGNER, R.; FRANCISCATTO, R.; PASSERINO, L. M.; DE LIMA, J. V.; SILVEIRA, S. R. SolAssist Learning: formação em tecnologias assistivas através de um MOOC e uma biblioteca virtual de soluções assistivas. Informática na Educação:

Teoria e Prática. Porto Alegre, v.20, n.2, mai./ago. 2017. Disponível em:

<https://seer.ufrgs.br/InfEducTeoriaPratica/article/view/65879/43623> Acesso em: mar. 2020.

ZHENG, S.; WISNIEWSKI, P.; ROSSON, M. B.; CARROLL, J. M. Ask the Instructors: Motivations and Challenges of Teaching Massive Open Online Courses. Proceedings ACM Conference on Computer Supported Cooperative Work \& Social Computing. p. 206-221. 2016. Disponível em: <https://dl.acm.org/doi/abs/10.1145/2818048.2820082> Acesso em: abr. 2020.

ZHOU, M. Chinese university students' acceptance of MOOCs: a self-determination perspective. Computers \& Education, vol 92-93, p. 194-203, jan./fev. 2016. Disponível em: <https://doi.org/10.1016/j.compedu.2015.10.012> Acesso em: abr. 2020. 